\title{
THE BERGMAN SPACES, THE BLOCH SPACE, AND GLEASON'S PROBLEM
}

\author{
KEHE ZHU
}

\begin{abstract}
Suppose $f$ is a holomorphic function on the open unit ball $B_{n}$ of $\mathbf{C}^{n}$. For $1 \leq p<\infty$ and $m>0$ an integer, we show that $f$ is in $L^{p}\left(B_{n}, d V\right)$ (with $d V$ the volume measure) iff all the functions $\partial^{m} f / \partial z^{\alpha}(|\alpha|=m)$ are in $L^{p}\left(B_{n}, d V\right)$. We also prove that $f$ is in the Bloch space of $B_{n}$ iff all the functions $\partial^{m} f / \partial z^{\alpha}(|\alpha|=m)$ are bounded on $B_{n}$. The corresponding result for the little Bloch space of $B_{n}$ is established as well. We will solve Gleason's problem for the Bergman spaces and the Bloch space of $B_{n}$ before proving the results stated above. The approach here is functional analytic. We make extensive use of the reproducing kernels of $B_{n}$. The corresponding results for the polydisc in $\mathbf{C}^{n}$ are indicated without detailed proof.
\end{abstract}

1. Introduction. Let $B_{n}$ be the open unit ball in $\mathbf{C}^{n}$ with $d V$ the normalized volume measure on $B_{n}$. The Bergman space $L_{a}^{p}\left(B_{n}\right)$ is the closed subspace of $L^{p}\left(B_{n}, d V\right)$ consisting of holomorphic functions. The Bloch space $\mathscr{B}\left(B_{n}\right)$ is the space of holomorphic functions $f$ on $B_{n}$ with the property that $\left(1-|z|^{2}\right)|\nabla f(z)|$ is bounded on $B_{n}$, where $\nabla f(z)=\left(\partial f(z) / \partial z_{1}, \ldots, \partial f(z) / \partial z_{n}\right)$ is the analytic gradient of $f$. It is clear that a holomorphic function $f$ on $B_{n}$ is in $\mathscr{B}\left(B_{n}\right)$ iff the functions

$$
\left(1-|z|^{2}\right) \frac{\partial f}{\partial z_{1}}(z), \ldots,\left(1-|z|^{2}\right) \frac{\partial f}{\partial z_{h}}(\text { ) }
$$

are in $L^{\infty}\left(B_{n}\right)$.

For $\alpha=\left(\alpha_{1}, \ldots, \alpha_{n}\right)$ with each $\alpha_{i}$ a nonnegative integer, we will write $|\alpha|=\alpha_{1}+$ $\cdots+\alpha_{n}, z^{\alpha}=z_{1}^{\alpha_{1}} \cdots z_{n}^{\alpha_{n}}, \overline{w^{\alpha}}=\bar{w}_{1}^{\alpha_{1}} \cdots \bar{w}_{n}^{\alpha_{n}}$, and $\partial^{|\alpha|} f(z) / \partial z^{\alpha}=\partial^{|\alpha|} f(z) / \partial z_{1}^{\alpha_{1}}$ $\cdots \partial z_{n}^{\alpha_{n}}$.

The main results of this paper are Theorems A, B, and C.

THEOREM A. Let $m$ be a positive integer and $f$ be a holomorphic function on $B_{n}$. Then

(1) $f \in L_{a}^{p}\left(B_{n}\right)(1 \leq p<+\infty)$ iff the functions $\left(1-|z|^{2}\right)^{m} \partial^{m} f(z) / \partial z^{\alpha}(|\alpha|=m)$ are in $L^{p}\left(B_{n}, d V\right)$;

(2) $f \in \mathscr{B}\left(B_{n}\right)$ iff the functions $\left(1-|z|^{2}\right)^{m} \partial^{m} f(z) / \partial z^{\alpha}(|\alpha|=m)$ are in $L^{\infty}\left(B_{n}, d V\right)$.

When $n=1$, we will denote by $\mathbf{D}$ the open unit disc in $\mathbf{C}$ and $d A$ the normalized area measure on $\mathbf{D}$. We state the results in Theorem $\mathrm{A}$ for $n=1$ as a corollary.

Received by the editors June 15, 1987.

1980 Mathematics Subject Classification (1985 Revision). Primary 32A10, 46E15; Secondary 32H10, 47B38. 
COROLlaRY. Suppose $m$ is a positive integer and $f$ is analytic on $\mathbf{D}$. Then

(1) $f \in L_{a}^{p}(\mathbf{D})(1 \leq p<\infty)$ iff $\left(1-|z|^{2}\right)^{m} f^{(m)}(z)$ is in $L^{p}(\mathbf{D}, d A)$;

(2) $f \in \mathscr{B}(\mathbf{D})$ iff $\left(1-|z|^{2}\right)^{m} f^{(m)}(z)$ is in $L^{\infty}(\mathbf{D}, d A)$.

Part (1) in the above corollary is due to Hardy, Littlewood, and Zabulionsis, see [6] and Theorem 5.6 of [3]. Part (2) in the above corollary is well known (see [1] or Theorem 5.5 of [3], for example). Thus Theorem A is a generalization of these known results on the disc to the open unit ball $B_{n}$. In particular, Theorem A partially answers a question posed at the end of $\S 2$ in [2]. Theorem A exhibits a special connection between the Bloch space and the Bergman spaces. We can think of the Bloch space $\mathscr{B}\left(B_{n}\right)$ as the Bergman space $L_{a}^{p}\left(B_{n}\right)$ as $p \rightarrow+\infty$. This property is unique for the balls among bounded symmetric domains. For the polydisc $\mathbf{D}^{n}$ in $\mathbf{C}^{n}$, the "limit" of the Bergman spaces $L_{a}^{p}\left(\mathbf{D}^{n}\right)$ as $p \rightarrow \infty$ is strictly larger than the Bloch space. We state the result for the bidisc.

THEOREM B. Suppose $f$ is holomorphic on $\mathbf{D}^{2}$, and $1 \leq p<+\infty$. Then $f \in L_{a}^{p}\left(\mathbf{D}^{2}\right)$ iff the functions

$\left(1-\left|z_{1}\right|^{2}\right) \frac{\partial f}{\partial z_{1}}\left(z_{1}, 0\right), \quad\left(1-\left|z_{2}\right|^{2}\right) \frac{\partial f}{\partial z_{2}}\left(0, z_{2}\right), \quad\left(1-\left|z_{1}\right|^{2}\right)\left(1-\left|z_{2}\right|^{2}\right) \frac{\partial^{2} f}{\partial z_{1} \partial z_{2}}\left(z_{1}, z_{2}\right)$

are in $L^{p}\left(\mathrm{D}^{2}, d V\right)$.

The space of holomorphic functions $f$ on $\mathbf{D}^{2}$ with the above three functions being in $L^{\infty}\left(\mathbf{D}^{2}\right)$ strictly contains the Bloch space $\mathscr{B}\left(\mathbf{D}^{2}\right)[\mathbf{7}]$.

The main tools that we need in this paper are:

(1) The boundedness of a family of projections from $L^{p}\left(B_{n}, d V\right)$ onto $L_{a}^{p}\left(B_{n}\right)$ for all $p \in[1,+\infty)$ (see [4]);

(2) The generalized Gleason's problem for the Bergman spaces and the Bloch space, which we state as

THEOREM C. For any $m \geq 1$, there exist linear operators $A_{\alpha}(|\alpha|=m)$ on holomorphic functions in $B_{n}$ such that

(1) If $f$ and all its partial derivatives of order $\leq m-1$ are zero at 0 , then

$$
f(z)=\sum_{|\alpha|=m} z^{\alpha} A_{\alpha} f(z), \quad z \in B_{n}
$$

(2) Each $A_{\alpha}$ is bounded on $L_{a}^{p}\left(B_{n}\right)$ for all $1 \leq p<\infty$;

(3) Each $A_{\alpha}$ is bounded on $\mathscr{B}\left(B_{n}\right)$.

We study the generalized Gleason's problem in $\S 2$. $\S 3$ is devoted to the proof of part (1) of Theorem A when $1<p<+\infty$. Part (2) of Theorem A is proved in $\S 4$. The case $p=1$ is treated in $\S 5$. We outline the proof for Theorem B in $\S 6$.

The author wishes to thank Sheldon Axler and Karel Stroethoff for pointing out the reference [6]. The author is grateful to the referee for some very useful comments and simplifying the proof of Theorems 5 and 6 . The referee also pointed out that some of the reproducing kernel arguments could be handled alternatively by integration by parts. 
2. Gleason's problem. Let $X$ be a space of holomorphic functions on a domain $\Omega$ in $\mathbf{C}^{n}$. Then Gleason's problem for $X$ is the following: Given $a \in \Omega, f \in X$, and $f(a)=0$, do there exist functions $f_{1}, \ldots, f_{n}$ in $X$ such that

$$
f(z)=\sum_{k=1}^{n}\left(z_{k}-a_{k}\right) f_{k}(z)
$$

for all $z$ in $\Omega$ ?

The difficulty of this problem depends on $\Omega$ and $X$. Gleason originally asked the question for $\Omega=B_{n}, a=0$, and $X=$ the ball algebra $A\left(B_{n}\right)$ (the space of holomorphic functions in $B_{n}$ which are continuous on $\bar{B}_{n}$ ). Gleason's problem is trivial if $\Omega=\mathbf{D}^{n}$ and $a=0$. Gleason's original problem was solved by Leibenson, who observed that

$$
f(z)-f(0)=\sum_{k=1}^{n} z_{k} \int_{0}^{1} \frac{\partial f}{\partial z_{k}}(t z) d t
$$

and then proved that each function

$$
f_{k}(z)=\int_{0}^{1} \frac{\partial f}{\partial z_{k}}(t z) d t
$$

is in $A\left(B_{n}\right)$ if $f$ is in $A\left(B_{n}\right)$. For more information on this problem, see 6.6 of [5].

We will use Leibenson's idea and a family of bounded projections given by Forelli and Rudin to solve a generalized version of Gleason's problem on $B_{n}$ with $X$ being the Bergman spaces or the Bloch space.

For any $r \geq 0$, let $P_{r}$ be the operator defined by

$$
P_{r} f(z)=\lambda_{r} \int_{B_{n}} \frac{\left(1-|w|^{2}\right)^{r}}{(1-\langle z, w\rangle)^{n+1+r}} f(w) d V(w),
$$

where

$$
\lambda_{r}^{-1}=\int_{B_{n}}\left(1-|w|^{2}\right)^{r} d V(w)
$$

When $r=0, P_{r}$ is just the Bergman projection of $B_{n}$, or the orthogonal projection from the Hilbert space $L^{2}\left(B_{n}, d V\right)$ onto the closed subspace $L_{a}^{2}\left(B_{n}\right)$. When $r=m$ is a positive integer, we have

$$
\lambda_{m}=(n+m) ! / n ! m ! .
$$

In particular, $\lambda_{1}=n+1$.

LEMMA 1. When $r>0, P_{r}$ is a (nonselfadjoint) bounded projection from $L^{p}\left(B_{n}, d V\right)$ onto $L_{a}^{p}\left(B_{n}\right)$ for all $1 \leq p<\infty$. When $r=0, P_{r}$ is a selfadjoint bounded projection from $L^{p}\left(B_{n}, d V\right)$ onto $L_{a}^{p}\left(B_{n}\right)$ for all $1<p<+\infty$.

ProOF. This is a special case of Theorem 7.1.4 of [5]. Also see [4].

REMARK. The proof of Theorem 7.1.4 of [5] actually gives us a little more, that is, there exists a constant $C$ (depending only on $r$ and $p$ ) such that for all $f$ in $L^{p}\left(B_{n}, d V\right)$ and

$$
F(z)=\lambda_{r} \int_{B_{n}} \frac{\left(1-|w|^{2}\right)^{r}}{|1-\langle z, w\rangle|^{n+1+r}}|f(w)| d V(w)
$$

we have $\|F\|_{p} \leq C\|f\|_{p}(1 \leq p<\infty$ if $r>0$ and $1<p<\infty$ if $r=0)$. We will need this fact later.

An immediate consequence of Lemma 1 is the following. 
COROLlaRY 2. If $r>0$, then $P_{r}^{*}$ is bounded on $L^{p}\left(B_{n}, d V\right)$ for all $1<p \leq$ $+\infty$, where $P_{r}^{*}$ is given by

$$
P_{r}^{*} f(z)=\lambda_{r}\left(1-|z|^{2}\right)^{r} \int_{B_{n}} \frac{f(w) d V(w)}{(1-\langle z, w\rangle)^{n+1+r}} .
$$

We now look at Gleason's problem for the Bergman spaces and the Bloch space on $B_{n}$. To illustrate the ideas, we first solve the first-order Gleason's problem for the Bergman spaces and the Bloch space; then we will turn to higher order derivatives.

THEOREM 3. For any $1 \leq p<+\infty$, there exist bounded linear operators $A_{1}, \ldots, A_{n}$ on $L_{a}^{p}\left(B_{n}\right)$ such that

$$
f(z)=\sum_{k=1}^{n} z_{k} A_{k} f(z)
$$

for all $z$ in $B_{n}$ and $f$ in $L_{a}^{p}\left(B_{n}\right)$ with $f(0)=0$.

ProOF. Let $A_{k}(1 \leq k \leq n)$ be defined by

$$
A_{k} f(z)=\int_{0}^{1} \frac{\partial f}{\partial z_{k}}(t z) d t .
$$

Then for any holomorphic function $f$ on $B_{n}$, we have

$$
f(z)-f(0)=\sum_{k=1}^{n} z_{k} A_{k} f(z), \quad z \in B_{n} .
$$

$A_{k}$ is obviously linear, so it remains to show that $A_{k}$ is bounded on $L_{a}^{p}\left(B_{n}\right)$ for $1 \leq p<+\infty$.

Given $f \in L_{a}^{p}\left(B_{n}\right)$, by Lemma 1 , we can write

$$
f(z)=(n+1) \int_{B_{n}} \frac{\left(1-|w|^{2}\right) f(w) d V(w)}{(1-\langle z, w\rangle)^{n+2}} .
$$

Differentiating under the integral, we get

$$
\frac{\partial f}{\partial z_{k}}(z)=(n+1)(n+2) \int_{B_{n}} \frac{\bar{w}_{k}\left(1-|w|^{2}\right) f(w)}{(1-\langle z, w\rangle)^{n+3}} d V(w)
$$

and so

$$
\begin{aligned}
A_{k} f(z) & =\int_{0}^{1} \frac{\partial f}{\partial z_{k}}(t z) d t=(n+1)(n+2) \int_{0}^{1} d t \int_{B_{n}} \frac{\bar{w}_{k}\left(1-|w|^{2}\right) f(w)}{(1-t\langle z, w\rangle)^{n+3}} d V(w) \\
& =(n+1)(n+2) \int_{B_{n}} \bar{w}_{k}\left(1-|w|^{2}\right) f(w) d V(w) \int_{0}^{1} \frac{d t}{(1-t\langle z, w\rangle)^{n+3}} \\
& =(n+1) \int_{B_{n}} \frac{\bar{w}_{k}\left(1-|w|^{2}\right) f(w)}{(1-\langle z, w\rangle)^{n+2}} \cdot \frac{1-(1-\langle z, w\rangle)^{n+2}}{\langle z, w\rangle} d V(w) .
\end{aligned}
$$

Note that

$$
\frac{1-(1-\langle z, w\rangle)^{n+2}}{\langle z, w\rangle}
$$


is a polynomial in $z$ and $\bar{w}$. Thus we can find a constant $C>0$ such that

$$
\left|A_{k} f(z)\right| \leq C(n+1) \int_{B_{n}} \frac{\left(1-|w|^{2}\right)|f(w)|}{|1-\langle z, w\rangle|^{n+2}} d V(w) .
$$

By the remark after Lemma 1 , we see that $A_{k}$ is bounded on $L_{a}^{p}\left(B_{n}\right)$ for all $1 \leq$ $p<+\infty$.

Recall that the Bloch space $\mathscr{B}\left(B_{n}\right)$ consists of holomorphic functions $f$ with the property that $\left(1-|z|^{2}\right)|\nabla f(z)|$ is bounded on $B_{n}$, where $\nabla f(z)=\left(\partial f(z) / \partial z_{1}, \ldots\right.$, $\left.\partial f(z) / \partial z_{n}\right)$ is the analytic gradient of $f$. Define a norm \|\|$_{\mathscr{B}}$ on $\mathscr{B}\left(B_{n}\right)$ by

$$
\|f\|_{\mathscr{B}}=|f(0)|+\sup _{z \in B_{n}}\left(1-|z|^{2}\right)|\nabla f(z)| .
$$

Then $\mathscr{B}\left(B_{n}\right)$ becomes a Banach space. Note that $\|f\|_{\mathscr{B}}$ is equivalent to $|f(0)|+$ $\sum_{k=1}^{n} \sup _{z \in B_{n}}\left(1-|z|^{2}\right)\left|\partial f(z) / \partial z_{k}\right|$.

The little Bloch space $\mathscr{B}_{0}\left(B_{n}\right)$ is the subspace of $\mathscr{B}\left(B_{n}\right)$ consisting of functions $f$ with $\left(1-|z|^{2}\right)|\nabla f(z)| \rightarrow 0$ as $|z| \rightarrow 1^{-}$. It is well known that $\mathscr{B}_{0}\left(B_{n}\right)$ is the closure of the polynomials in $\mathscr{B}\left(B_{n}\right)$. If $P$ is the Bergman projection and $\mathbf{C}\left(\bar{B}_{n}\right)$ is the space of continuous complex functions on $\bar{B}_{n}$, then we have $\mathscr{B}\left(B_{n}\right)=P L^{\infty}\left(B_{n}\right)$, $\mathscr{B}_{0}\left(B_{n}\right)=P C\left(\bar{B}_{n}\right)$. See $[7]$.

THEOREM 4. There exist bounded linear operators $A_{1}, \ldots, A_{n}$ on $\mathscr{B}\left(B_{n}\right)$ such that

$$
f(z)-f(0)=\sum_{k=1}^{n} z_{k} A_{k} f(z)
$$

for all $f \in \mathscr{B}\left(B_{n}\right)$ and $z \in B_{n}$. Moreover, each $A_{k}$ leaves the little Bloch space $\mathscr{B}_{0}\left(B_{n}\right)$ invariant.

PROOF. Let $A_{k}$ be the operators defined in the proof of Theorem 3. We must prove that each $A_{k}$ maps $\mathscr{B}\left(B_{n}\right)$ (or $\mathscr{B}_{0}\left(B_{n}\right)$ ) into $\mathscr{B}\left(B_{n}\right)$ (or $\mathscr{B}_{0}\left(B_{n}\right)$ ) boundedly. We prove the result for $\mathscr{B}\left(B_{n}\right)$. A similar proof can be given to $\mathscr{B}_{0}\left(B_{n}\right)$ using the equality $\mathscr{B}_{0}\left(B_{n}\right)=P C\left(\bar{B}_{n}\right)$.

Given $f \in \mathscr{B}\left(B_{n}\right)$, we can find a function $\varphi \in L^{\infty}\left(B_{n}\right)$ such that $f=P \varphi$ and $C^{-1}\|f\|_{\mathscr{B}} \leq\|\varphi\|_{\infty} \leq C\|f\|_{\mathscr{B}}$, where $C$ is some positive constant independent of $f$. (The function $\varphi$ can even be explicitly written down, see [7].) Now

$$
f(z)=\int_{B_{n}} \frac{\varphi(w) d V(w)}{(1-\langle z, w\rangle)^{n+1}}
$$

and differentiating under the integral gives

$$
\frac{\partial f}{\partial z_{k}}(z)=(n+1) \int_{B_{n}} \frac{\bar{w}_{k} \varphi(w) d V(w)}{(1-\langle z, w\rangle)^{n+2}} .
$$

This implies that (by the proof of Theorem 3)

$$
A_{k} f(z)=\int_{0}^{1} \frac{\partial f}{\partial z_{k}}(t z) d t=\int_{B_{n}} \frac{\bar{w}_{k} \varphi(w)}{(1-\langle z, w\rangle)^{n+1}} Q(z, w) d V(w),
$$

where $Q(z, w)$ is the polynomial (in $z$ and $\bar{w}$ )

$$
Q(z, w)=\frac{1-(1-\langle z, w\rangle)^{n+1}}{\langle z, w\rangle}=\sum_{k=0}^{n}(1-\langle z, w\rangle)^{k}
$$


Write

$$
Q(z, w)=\sum a_{\alpha, \beta} z^{\alpha} \bar{w}^{\beta} \quad \text { (finite sum) }
$$

Then

$$
A_{k} f(z)=\sum a_{\alpha, \beta} z^{\alpha} \int_{B_{n}} \frac{\bar{w}_{k} \bar{w}^{\beta} \varphi(w)}{(1-\langle z, w\rangle)^{n+1}} d V(w)
$$

Since each function $\bar{w}_{k} \bar{w}^{\beta} \varphi(w)$ is still in $L^{\infty}\left(B_{n}\right)$ with sup-norm $\leq\|\varphi\|_{\infty}$, and $\mathscr{B}\left(B_{n}\right)=P L^{\infty}\left(B_{n}\right)$, each function

$$
\int_{B_{n}} \frac{\bar{w}_{k} \bar{w}^{\beta} \varphi(w)}{(1-\langle z, w\rangle)^{n+1}} d V(w)
$$

is in $\mathscr{B}\left(B_{n}\right)$ (as a function in $z$ ). Moreover, its norm in $\mathscr{B}\left(B_{n}\right)$ is at most a constant multiple of the norm of $f$ in $\mathscr{B}\left(B_{n}\right)$ (by the inequality $C^{-1}\|f\|_{\mathscr{B}} \leq$ $\left.\|\varphi\|_{\infty} \leq C\|f\|_{\mathscr{B}}\right)$. It is also easy to see that multiplication by $z^{\alpha}$ is a bounded operator on $\mathscr{B}\left(B_{n}\right)$. Therefore, $A_{k}$ is a bounded linear mapping on $\mathscr{B}\left(B_{n}\right)$ for all $1 \leq k \leq n$. This completes the proof of Theorem 4 .

Next we generalize Theorems 3 and 4 to higher orders.

THEOREM 5. Suppose $m \geq 1$ and $1 \leq p<+\infty$. Then for each $\alpha=\left(\alpha_{1}, \ldots, \alpha_{n}\right)$ with $|\alpha|=m$ there exists a bounded linear operator $A_{\alpha}$ on $L_{a}^{p}\left(B_{n}\right)$ such that if $f$ and all its partial derivatives of order $\leq m-1$ are zero at 0 , then

$$
f(z)=\sum_{|\alpha|=m} z^{\alpha} A_{\alpha} f(z)
$$

for all $z$ in $B_{n}$.

PROOF. For simplicity of notation, we prove the theorem for $m=2$ (the case $m=1$ is just Theorem 3). Given $f$ holomorphic in $B_{n}$, by Theorem 3 , we have

$$
f(z)=f(0)+\sum_{i=1}^{n} z_{i} A_{i} f(z) \text {. }
$$

It is easy to see that

$$
\frac{\partial f}{\partial z_{i}}(0)=A_{i} f(0) \quad(1 \leq i \leq n) .
$$

Now applying Theorem 3 to each of the functions $A_{i} f$, we get

$$
\begin{aligned}
f(z) & =f(0)+\sum_{i=1}^{n} z_{i}\left(\frac{\partial f}{\partial z_{i}}(0)+\sum_{j=1}^{n} z_{j} A_{j} A_{i} f(z)\right) \\
& =f(0)+\sum_{i=1}^{n} \frac{\partial f}{\partial z_{i}}(0) z_{i}+\sum_{i, j=1}^{n} z_{i} z_{j} A_{i} A_{j} f(z) .
\end{aligned}
$$

Let $A_{i j}=A_{i} A_{j}$; then by Theorem $3, A_{i j}$ is bounded on all $L_{a}^{p}\left(B_{n}\right)(1 \leq p<+\infty)$. Moreover, if $f(0)$ and all the first-order partial derivatives $\left(\partial f / \partial z_{i}\right)(0)$ are zero, then

$$
f(z)=\sum_{i, j=1}^{n} A_{i j} f(z) .
$$

This completes the proof of Theorem 5 .

Similarly, we can prove the following generalization of Theorem 4. 
THEOREM 6. Suppose $m \geq 1$. Then for each $\alpha=\left(\alpha_{1}, \ldots, \alpha_{n}\right)$ with $|\alpha|=m$, there exists a bounded linear operator $A_{\alpha}$ on $\mathscr{B}\left(B_{n}\right)$ such that if $f$ and all its partial derivatives of order $\leq m-1$ are zero at 0 , then

$$
f(z)=\sum_{|\alpha|=m} z^{\alpha} A_{\alpha} f(z)
$$

for all $z$ in $B_{n}$. Moreover, each $A_{\alpha}$ maps $\mathscr{B}_{0}\left(B_{n}\right)$ into $\mathscr{B}_{0}\left(B_{n}\right)$.

3. Characterizations of $L_{a}^{p}\left(B_{n}\right)$ for $1<p<\infty$. For any $m \geq 1$ and $|\alpha|=m$, we define the following operators:

$$
\begin{aligned}
& S_{\alpha} f(z)=\bar{z}^{\alpha} f(z), \quad T_{\alpha} f(z)=\left(1-|z|^{2}\right)^{m} \frac{\partial^{m} f}{\partial z^{\alpha}}(z), \\
& P_{m} f(z)=\lambda_{m} \int_{B_{n}} \frac{\left(1-|w|^{2}\right)^{m}}{(1-\langle z, w\rangle)^{n+m+1}} f(w) d V(w) .
\end{aligned}
$$

$S_{\alpha}$ and $P_{m}$ are well-defined for all functions $f$ in $L^{1}\left(B_{n}, d V\right)$, while $T_{\alpha}$ is only defined for holomorphic functions on $B_{n}$.

LEMMA 7. If $1<p<\infty$ and $f \in L_{a}^{p}\left(B_{n}\right)$, then for all $m \geq 1$ and $|\alpha|=m$, the functions $T_{\alpha} f(z)=\left(1-|z|^{2}\right)^{m} \partial^{m} f(z) / \partial z^{\alpha}$ are in $L^{p}\left(B_{n}, d V\right)$. Moreover, there is a constant $C>0$ such that $\left\|T_{\alpha} f\right\|_{p} \leq C\|f\|_{p}$ for all $f \in L_{a}^{p}\left(B_{n}\right)$.

PROOF. Given $f \in L_{a}^{p}\left(B_{n}\right)$ with $1<p<\infty$, we can write

$$
f(z)=\int_{B_{n}} \frac{f(w) d V(w)}{(1-\langle z, w\rangle)^{n+1}}
$$

Differentiating under the integral gives

$$
\frac{\partial^{m} f}{\partial z^{\alpha}}(z)=(n+1) \cdots(n+m) \int_{B_{n}} \frac{\bar{w}^{\alpha} f(w)}{(1-\langle z, w\rangle)^{n+m+1}} d V(w) .
$$

This implies that

$$
\begin{aligned}
T_{\alpha} f(z) & =\left(1-|z|^{2}\right)^{m} \frac{\partial^{m} f}{\partial z^{\alpha}}(z) \\
& =(n+1) \cdots(n+m)\left(1-|z|^{2}\right)^{m} \int_{B_{n}} \frac{S_{\alpha} f(w) d V(w)}{(1-\langle z, w\rangle)^{n+m+1}} \\
& =\frac{(n+1) \cdots(n+m)}{\lambda_{m}} P_{m}^{*} S_{\alpha} f(z)=m ! P_{m}^{*} S_{\alpha} f(z) .
\end{aligned}
$$

Since $S_{\alpha}$ and $P_{m}^{*}$ are bounded operators on $L^{p}\left(B_{n}, d V\right)$ for all $1<p<+\infty$, thus we have

This proves Lemma 7 .

$$
\left\|T_{\alpha} f\right\|_{p} \leq m !\left\|P_{m}^{*} S_{\alpha}\right\|_{p}\|f\|_{p}
$$

The next three lemmas will establish the converse of Lemma 7.

LEMMA 8. Let $1 \leq p<+\infty$ and $m \geq 1$. Then there exists a constant $C>0$ such that every function $f$ in $L_{a}^{p}\left(B_{n}\right)$ can be written as

$$
f(z)=f_{0}(z)+\sum_{|\alpha|=m} z^{\alpha} f_{\alpha}(z)
$$

with $f_{0}$ a polynomial of order $\leq m-1$ and $\left\|f_{0}\right\|_{p} \leq C\|f\|_{p},\left\|f_{\alpha}\right\| \leq C\|f\|_{p}$. 
Proof. Let $f_{0}(z)$ be the $(m-1)$ th order Taylor polynomial of $f$ at 0 . Let $g=f-f_{0}$. Then $g$ and all its partial derivatives of order $\leq m-1$ are zero at 0 . By Theorem 5, there are functions $f_{\alpha}$ in $L_{a}^{p}\left(B_{n}\right)$ such that

$$
g(z)=\sum_{|\alpha|=m} z^{\alpha} f_{\alpha}(z)
$$

and $\left\|f_{\alpha}\right\|_{p} \leq C_{1}\|g\|_{p}$ for all $|\alpha|=m$, where $C_{1}$ is a constant independent of $f$. So it remains to show that $\left\|f_{0}\right\|_{p} \leq C_{2}\|f\|_{p}$ for some other constant $C_{2}>0$.

Given $f$ in $L_{a}^{p}\left(B_{n}\right)$, write $f=P f$ and differentiate under the integral; we get

$$
\frac{\partial^{|\beta|} f}{\partial z^{\beta}}(0)=(n+1) \cdots(n+|\beta|) \int_{B_{n}} \bar{w}^{\beta} f(w) d V(w) .
$$

This implies that

$$
\left|\frac{\partial^{|\beta|} f}{\partial z^{\beta}}(0)\right| \leq(n+1) \cdots(n+|\beta|)\|f\|_{p} \quad(1 \leq p<+\infty) .
$$

Since $f_{0}(z)$ is a polynomial of order $\leq m-1$ with coefficients linearly depending on $\left(\partial^{|\beta|} f / \partial z^{\beta}\right)(0)$, we must have $\left\|f_{0}\right\|_{p} \leq C_{2}\|f\|_{p}$ for some constant $C_{2}>0$ independent of $f$. This finishes the proof of Lemma 8.

For any $m \geq 1,1 \leq p<+\infty$, and $f$ holomorphic in $B_{n}$, let

$$
\|f\|_{m, p}=\left\|f_{0}\right\|_{p}+\sum_{|\alpha|=m}\left\|T_{\alpha} f\right\|_{p}
$$

where $f(z)=f_{0}(z)+\sum_{|\alpha|=m} z^{\alpha} f_{\alpha}(z)$ is the decomposition given in the proof of Lemma 8. By Lemmas 7 and $8,\|\|_{m, p}$ is a well-defined norm on $L_{a}^{p}\left(B_{n}\right)$ for $1<p<+\infty$. Moreover, there exists a constant $C>0$ such that $\|f\|_{m, p} \leq C\|f\|_{p}$ for all $f$ in $L_{a}^{p}\left(B_{n}\right)$. Note that

$$
\left\|f_{0}\right\|_{p} \sim \sum_{|\beta| \leq m-1}\left|\frac{\partial^{|\beta|} f}{\partial z^{\beta}}(0)\right| .
$$

Thus

$$
\|f\|_{m, p} \sim \sum_{|\beta| \leq m-1}\left|\frac{\partial^{|\beta|} f}{\partial z^{\beta}}(0)\right|+\sum_{|\alpha|=m}\left\|T_{\alpha} f\right\|_{p} .
$$

LEMma 9. Suppose $1<p<+\infty$. Then there exists a constant $C>0$ such that $\|f\|_{p} \leq C\|f\|_{m, p}$ for all $f \in L_{a}^{p}\left(B_{n}\right)$.

Proof. Given $f \in L_{a}^{p}\left(B_{n}\right)$, then by the duality $L_{a}^{p}\left(B_{n}\right)^{*} \cong L_{a}^{q}\left(B_{n}\right)\left(\frac{1}{p}+\frac{1}{q}=1\right)$, we have

$$
\|f\|_{p} \leq C_{1} \sup \left\{|\langle f, g\rangle|: g \in L_{a}^{q}\left(B_{n}\right),\|g\|_{q}=1\right\},
$$

where $C_{1}$ is a constant and the pairing $\langle$,$\rangle is given by$

$$
\langle f, g\rangle=\int_{B_{n}} f(z) \overline{g(z)} d V(z) .
$$


For any $g \in L_{a}^{q}\left(B_{n}\right)$, by Lemma 8 , we can write

$$
g(z)=g_{0}(z)+\sum_{|\alpha|=m} z^{\alpha} g_{\alpha}(z)
$$

or

$$
g=g_{0}+\sum_{|\alpha|=m} S_{\alpha}^{*} g_{\alpha}
$$

with $\left\|g_{0}\right\|_{q} \leq C_{2}\|g\|_{q},\left\|g_{\alpha}\right\|_{q} \leq C_{2}\|g\|_{q}$ for some constant $C_{2}>0$. Thus we have

$$
\begin{aligned}
\langle f, g\rangle & =\left\langle f, g_{0}\right\rangle+\sum_{|\alpha|=m}\left\langle f, S_{\alpha}^{*} g_{\alpha}\right\rangle \\
& =\left\langle f_{0}, g_{0}\right\rangle+\sum_{|\alpha|=m}\left\langle S_{\alpha} f, g_{\alpha}\right\rangle \\
& =\left\langle f_{0}, g_{0}\right\rangle+\sum_{|\alpha|=m}\left\langle S_{\alpha} f, P_{m} g_{\alpha}\right\rangle \\
& =\left\langle f_{0}, g_{0}\right\rangle+\sum_{|\alpha|=m}\left\langle P_{m}^{*} S_{\alpha} f, g_{\alpha}\right\rangle \\
& =\left\langle f_{0}, g_{0}\right\rangle+\frac{1}{m !} \sum_{|\alpha|=m}\left\langle T_{\alpha} f, g_{\alpha}\right\rangle .
\end{aligned}
$$

This implies thai

$$
\begin{aligned}
|\langle f, g\rangle| & \leq\left\|f_{0}\right\|_{p}\left\|g_{0}\right\|_{q}+\frac{1}{m !} \sum_{|\alpha|=m}\left\|T_{\alpha} f\right\|_{p}\left\|g_{\alpha}\right\|_{q} \\
& \leq\left[\left\|f_{0}\right\|_{p}+\frac{1}{m !} \sum_{|\alpha|=m}\left\|T_{\alpha} f\right\|_{p}\right] C_{2}\|g\|_{q} \leq C_{2}\|f\|_{m, p}\|g\|_{q} .
\end{aligned}
$$

Hence

$$
\begin{aligned}
\|f\|_{p} & \leq C_{1} \sup \left\{|\langle f, g\rangle|: g \in L_{a}^{p}\left(B_{n}\right),\|g\|_{q}=1\right\} \\
& \leq C_{1} C_{2}\|f\|_{m, p}
\end{aligned}
$$

This completes the proof of Lemma 9.

REMARK. Lemmas 7 and 9 imply that \|\|$_{p}$ and \|\|$_{m, p}$ are equivalent norms on $L_{a}^{p}\left(B_{n}\right)$ for all $1<p<+\infty$ and $m \geq 1$. However, they do not say that $\|f\|_{m, p}<+\infty$ implies $\|f\|_{p}<+\infty$. We needed the assumption $f \in L_{a}^{p}\left(B_{n}\right)$ to justify the "inner product" $\langle f, g\rangle$ and the applicability of all the operators $P_{m}, P_{m}^{*}$, $S_{\alpha}, S_{\alpha}^{*}$. The following lemma overcomes this difficulty.

LEMMA 10. Suppose $m \geq 1,1<p<+\infty$, and $f$ is holomorphic in $B_{n}$. Then $\|f\|_{m, p}<+\infty$ implies $f \in L_{a}^{p}\left(B_{n}\right)$. 
PROOF. Let $f_{r}(z)=f(r z), r \in(0,1)$. Then $f_{r}$ is in $L_{a}^{p}\left(B_{n}\right)$ for all $1<p<+\infty$. Moreover, for $|\alpha|=m$,

$$
\begin{aligned}
\int_{B_{n}}\left|T_{\alpha} f_{r}(z)\right|^{p} d V(z) & =\int_{B_{n}}\left(1-|z|^{2}\right)^{m p}\left|\frac{\partial^{m} f_{r}}{\partial z^{\alpha}}(z)\right|^{p} d V(z) \\
& =\int_{B_{n}} r^{m p}\left(1-|z|^{2}\right)^{m p}\left|\frac{\partial^{m} f}{\partial z^{\alpha}}(r z)\right|^{p} d V(z) \\
& \leq r^{m p} \int_{B_{n}}\left(1-r^{2}|z|^{2}\right)^{m p}\left|\frac{\partial^{m} f}{\partial z^{\alpha}}(r z)\right|^{p} d V(z) \\
& =\frac{r^{m p}}{r^{2 n}} \int_{|z|<r}\left(1-|z|^{2}\right)^{m p}\left|\frac{\partial^{m} f}{\partial z^{\alpha}}(z)\right|^{p} d V(z) \\
& \leq \frac{r^{m p}}{r^{2 n}} \int_{B_{n}}\left(1-|z|^{2}\right)^{m p}\left|\frac{\partial^{m} f}{\partial z^{\alpha}}(z)\right|^{p} d V(z) \\
& =\frac{1}{r^{2 n-m p}} \int_{B_{n}}\left|T_{\alpha} f(z)\right|^{p} d V(z) .
\end{aligned}
$$

Thus we can find a constant $C_{1}>0$ such that

$$
\left\|T_{\alpha} f_{r}\right\|_{p} \leq C_{1}\left\|T_{\alpha} f\right\|_{p}
$$

for all $r \in\left[\frac{1}{2}, 1\right)$. It is easy to find another constant $C_{2}>0$ such that

$$
\left\|f_{r}\right\|_{m, p} \leq C_{2}\|f\|_{m, p}
$$

for all $r \in\left[\frac{1}{2}, 1\right)$. By Lemma 9 ,

$$
\left\|f_{r}\right\|_{p} \leq C_{3}\|f\|_{m, p}
$$

for all $r \in\left[\frac{1}{2}, 1\right)$. Letting $r \rightarrow 1^{-}$, we get

$$
\|f\|_{p} \leq C_{3}\|f\|_{m, p}<+\infty .
$$

This proves that $f$ is in $L_{a}^{p}\left(B_{n}\right)$ if $\|f\|_{m, p}<+\infty$.

Summarizing Lemmas 6-10, we have proved the main result of this section.

THEOREM 11. Suppose $1<p<+\infty$ and $m \geq 1$ is an integer, then a holomorphic function $f$ on $B_{n}$ is in $L_{a}^{p}\left(B_{n}\right)$ iff all the functions $\left(1-|z|^{p}\right)^{m} \partial^{m} f(z) / \partial z^{\alpha}$ $(|\alpha|=m)$ are in $L^{p}\left(B_{n}, d V\right)$. Moreover, \|\|$_{p}$ and \|\|$_{m, p}$ are equivalent norms on $L_{a}^{p}\left(B_{n}\right)$.

COROLlaRY 12. Suppose $1<p<+\infty$ and $m \geq 1$. Then an analytic function $f$ on $\mathbf{D}$ is in $L_{a}^{p}(\mathbf{D})$ iff $\left(1-|z|^{2}\right)^{m} f^{(m)}(z)$ is in $L^{p}(\mathbf{D}, d V)$.

4. Characterizations of the Bloch space $\mathscr{B}\left(B_{n}\right)$. For $m \geq 1$ and $f$ holomorphic in $B_{n}$, let

$$
\|f\|_{m, \mathscr{B}}=\sum_{|\alpha| \leq m-1}\left|\frac{\partial^{|\alpha|} f}{\partial z^{\alpha}}(0)\right|+\sum_{|\alpha|=m} \sup _{z \in B_{n}}\left|\left(1-|z|^{2}\right)^{m} \frac{\partial^{m} f}{\partial z^{\alpha}}(z)\right| .
$$

The main result of this section is that $f$ belongs to $\mathscr{B}\left(B_{n}\right)$ iff $\|f\|_{m, \mathscr{B}}<+\infty$. Moreover, \|\|$_{\mathscr{B}}$ and \|\|$_{m, \mathscr{B}}$ are equivalent norms on $\mathscr{B}\left(B_{n}\right)$. A similar result will be proven for the little Bloch space $\mathscr{B}_{0}\left(B_{n}\right)$. 
LEMMA 13. For any $m \geq 1$, there exists a constant $C>0$ such that $\|f\|_{m, \mathscr{B}} \leq$ $C\|f\|_{\mathscr{B}}$ for all $f$ in $\mathscr{B}\left(B_{n}\right)$.

ProOF. By [7], there exists a constant $C_{1}>0$ such that for any $f \in \mathscr{B}\left(B_{n}\right)$, we can find $\varphi \in L^{\infty}\left(B_{n}\right)$ with $f=P \varphi$ and $\|\varphi\|_{\infty} \leq C_{1}\|f\|_{\mathscr{B}}$. Also there exists a constant $C_{2}>0$ such that $\|P \varphi\|_{\mathscr{B}} \leq C_{2}\|\varphi\|_{\infty}$ for all $\varphi$ in $L^{\infty}\left(B_{n}\right)$. Now suppose $f$ is in $\mathscr{B}\left(B_{n}\right)$. Choose $\varphi \in L^{\infty}\left(B_{n}\right)$ such that $f=P \varphi$ with $\|\varphi\|_{\infty} \leq C_{1}\|f\|_{\mathscr{B}}$. Write

$$
f(z)=\int_{B_{n}} \frac{\varphi(w) d V(w)}{(1-\langle z, w\rangle)^{n+1}}
$$

and differentiate under the integral. Then for any $|\alpha|=m$ we have

$$
\frac{\partial^{m} f}{\partial z^{\alpha}}(z)=(n+1) \cdots(n+m) \int_{B_{n}} \frac{\bar{w}^{\alpha} \varphi(w) d V(w)}{(1-\langle z, w\rangle)^{n+m+1}} .
$$

This implies that

$$
T_{\alpha} f(z)=\left(1-|z|^{2}\right)^{m} \frac{\partial^{m} f}{\partial z^{\alpha}}(z)=m ! P_{m}^{*} S_{\alpha} \varphi(z) .
$$

Since $S_{\alpha}$ and $P_{m}^{*}$ are bounded on $L^{\infty}\left(B_{n}\right)$ by Corollary 2, we have

$$
\left\|T_{\alpha} f\right\|_{\infty} \leq m !\left\|P_{m}^{*} S_{\alpha}\right\|_{\infty}\|\varphi\|_{\infty} \leq C_{1} m !\left\|P_{m}^{*} S_{\alpha}\right\|_{\infty}\|f\|_{\mathscr{B}}
$$

for all $\alpha=\left(\alpha_{1}, \ldots, \alpha_{n}\right)$ with $|\alpha|=m$. We also have

$$
\begin{aligned}
\left|\frac{\partial^{|\beta|} f}{\partial z^{\beta}}(0)\right| & =(n+1) \cdots(n+|\beta|)\left|\int_{B_{n}} \bar{w}^{\beta} \varphi(w) d V(w)\right| \\
& \leq(n+1) \cdots(n+|\beta|)\|\varphi\|_{\infty} \\
& \leq C_{1}(n+1) \cdots(n+|\beta|)\|f\|_{\mathscr{B}}
\end{aligned}
$$

for all $\beta=\left(\beta_{1}, \ldots, \beta_{n}\right) \geq 0$. Therefore, we can find a constant $C>0$ such that $\|f\|_{m, \mathscr{B}} \leq C\|f\|_{\mathscr{B}}$ for all $f$ in $\mathscr{B}\left(B_{n}\right)$. This completes the proof of Lemma 13.

LEMMA 14. Suppose $m \geq 1$, and $f$ is holomorphic in $B_{n}$. Then $\|f\|_{m, \mathscr{B}}<$ $+\infty$ implies that $f \in \mathscr{B}\left(B_{n}\right)$. Moreover, there exists a constant $C>0$ such that $\|f\|_{\mathscr{B}} \leq C\|f\|_{m, \mathscr{B}}$ for all $f$ in $\mathscr{B}\left(B_{n}\right)$.

Proof. Suppose $\|f\|_{m, \mathscr{B}}<+\infty$. Then it is clear that $\|f\|_{m, p}<+\infty$ for all $1 \leq p<+\infty$. By Lemma 10, we have $f \in L_{a}^{2}\left(B_{n}\right)$. Since $L_{a}^{1}\left(B_{n}\right)^{*} \cong \mathscr{B}\left(B_{n}\right)$ (see [7] for example) with the usual integral pairing, it suffices to show that $f$ induces a bounded linear functional on $L_{a}^{1}\left(B_{n}\right)$. Note that $L_{a}^{2}\left(B_{n}\right)$ is dense in $L_{a}^{1}\left(B_{n}\right)$, so it suffices to produce a constant $C>0$ such that $|\langle f, g\rangle|=\left|\int_{\Omega} f(z) \overline{g(z)} d V(z)\right| \leq$ $C\|f\|_{m, \mathscr{B}}\|g\|_{1}$ for all $g$ in $L_{a}^{2}\left(B_{n}\right)$. ( $C$ is independent of $f$ and $g$.)

Given $g \in L_{a}^{2}\left(B_{n}\right)$, write

$$
g=g_{0}+\sum_{|\alpha|=m} S_{\alpha}^{*} g_{\alpha}
$$


with $\left\|g_{0}\right\|_{1} \leq C_{1}\|g\|_{1},\left\|g_{\alpha}\right\|_{1} \leq C_{1}\|g\|_{1}$ (by Lemma 8) for some constant $C_{1}>0$ (independent of $g$ ). Now

$$
\begin{aligned}
\langle f, g\rangle & =\left\langle f, g_{0}\right\rangle+\sum_{|\alpha|=m}\left\langle f, S_{\alpha}^{*} g_{\alpha}\right\rangle \\
& =\left\langle f_{0}, g_{0}\right\rangle+\sum_{|\alpha|=m}\left\langle P_{m}^{*} S_{\alpha} f, g_{\alpha}\right\rangle \\
& =\left\langle f_{0}, g_{0}\right\rangle+\frac{1}{m !} \sum_{|\alpha|=m}\left\langle T_{\alpha} f, g_{\alpha}\right\rangle .
\end{aligned}
$$

It follows that

$$
|\langle f, g\rangle| \leq\left|\left\langle f_{0}, g_{0}\right\rangle\right|+\frac{1}{m !} \sum_{|\alpha|=m}\left\|T_{\alpha} f\right\|_{\infty}\left\|f_{\alpha}\right\|_{1} .
$$

It is also easy to see that

$$
\left|\left\langle f_{0}, g_{0}\right\rangle\right| \leq\left\|f_{0}\right\|_{\infty}\left\|g_{0}\right\|_{1} \leq C_{2}\left(\sum_{|\beta| \leq m-1}\left|\frac{\partial^{|\beta|} f}{\partial z^{\beta}}(0)\right|\right)\left\|g_{0}\right\|_{1} .
$$

Therefore, we can find a constant $C_{3}>0$ such that

$$
|\langle f, g\rangle| \leq C_{3}\|f\|_{m, \mathscr{B}}\|g\|_{1}
$$

for all $g$ in $L_{a}^{2}\left(B_{n}\right)$. This shows that $f \in \mathscr{B}\left(B_{n}\right)$ and $\|f\|_{\mathscr{B}} \leq C\|f\|_{m, \mathscr{B}}$ for some constant $C>0$ independent of $f$. This completes the proof of Lemma 4.

Combining Lemmas 13 and 14, we have proved the following

THEOREM 15. Given $m \geq 1$ and $f$ holomorphic in $B_{n}$, we have $f \in \mathscr{B}\left(B_{n}\right)$ iff the functions $\left(1-|z|^{2}\right)^{m} \partial^{m} f(z) / \partial z^{\alpha}(|\alpha|=m)$ are all in $L^{\infty}\left(B_{n}\right)$. Moreover, \|\|$_{\mathscr{B}}$ and \|\|$_{m, \mathscr{B}}$ are equivalent norms on $\mathscr{B}\left(B_{n}\right)$.

We prove a similar result for the little Bloch space $\mathscr{B}_{0}\left(B_{n}\right)$.

THEOREM 16. Given $m \geq 1$ and $f$ holomorphic in $B_{n}$, we have $f \in \mathscr{B}_{0}\left(B_{n}\right)$ iff all the functions $\left(1-|z|^{2}\right)^{m} \partial^{m} f(z) / \partial z^{\alpha}(|\alpha|=m)$ are in $\mathbf{C}_{0}\left(B_{n}\right)$, that is,

$$
\left(1-|z|^{2}\right)^{m} \partial^{m} f(z) / \partial z^{\alpha} \rightarrow 0 \quad\left(|z| \rightarrow 1^{-}\right)
$$

for all $|\alpha|=m$.

PROOF. If $f$ is a polynomial, then clearly $\left(1-|z|^{2}\right)^{m} \partial^{m} f(z) / \partial z^{\alpha} \rightarrow 0\left(|z| \rightarrow 1^{-}\right)$ for all $|\alpha|=m$. Since the little Bloch space $\mathscr{B}_{0}\left(B_{n}\right)$ is generated by the polynomials, and by Theorem 15 ,

$$
\sup _{z \in B_{n}}\left(1-|z|^{2}\right)^{m}\left|\frac{\partial^{m} f}{\partial z^{\alpha}}(z)\right| \leq C\|f\|_{\mathscr{B}},
$$

it follows that $\left(1-|z|^{2}\right)^{m} \partial^{m} f(z) / \partial z^{\alpha} \rightarrow 0\left(|z| \rightarrow 1^{-}\right)$for all $f$ in $\mathscr{B}_{0}\left(B_{n}\right)$ and $|\alpha|=m$.

Conversely, suppose $f \in \mathscr{B}\left(B_{n}\right)$ and $\left(1-|z|^{2}\right)^{m} \partial^{m} f(z) / \partial z^{\alpha} \rightarrow 0\left(|z| \rightarrow 1^{-}\right)$for all $|\alpha|=m$. Then it follows that

$$
\sup _{z \in B_{n}}\left(1-|z|^{2}\right)^{m}\left|\frac{\partial^{m} f}{\partial z^{\alpha}}(z)-\frac{\partial^{m} f_{r}}{\partial z^{\alpha}}(z)\right| \rightarrow 0 \quad\left(r \rightarrow 1^{-}\right),
$$


where $f_{r}(z)=f(r z)$. By Theorem 15, this implies $\left\|f-f_{r}\right\|_{\mathscr{B}} \rightarrow 0\left(r \rightarrow 1^{-}\right)$. Since $f_{r}$ is in $\mathscr{B}_{0}\left(B_{n}\right)$ and $\mathscr{B}_{0}\left(B_{n}\right)$ is closed in $\mathscr{B}\left(B_{n}\right)$. We must have $f \in \mathscr{B}_{0}\left(B_{n}\right)$. This proves Theorem 16.

5. Characterizations of the Bergman space $L_{a}^{1}\left(B_{n}\right)$. Let $m \geq 1$ and $f$ be a holomorphic function in $B_{n}$. We determined in $\S 3$ and $\S 4$ exactly when the functions $\left(1-|z|^{2}\right)^{m} \partial^{m} f(z) / \partial z^{\alpha}(|\alpha|=m)$ are in $L^{p}\left(B_{n}, d V\right)$ for $1<p \leq+\infty$. We settle the case $p=1$ in this section.

LEMMA 17. There exists a constant $C>0$ such that

$$
\int_{B_{n}} \frac{\left(1-|z|^{2}\right)^{m}}{|1-\langle z, w\rangle|^{n+m+2}} d V(z) \leq \frac{C}{1-|w|^{2}}
$$

for all $w$ in $B_{n}$.

PROOF. For any $a \in B_{n}$, there exists a biholomorphic mapping $\varphi_{a}$ of $B_{n}$ with the following properties:

(1) $\varphi_{a}(0)=a, \varphi_{a}(a)=0$;

(2) $\varphi_{a}^{2}=\mathrm{Id}$;

(3) The real Jacobian of $\varphi_{a}$ is $\left(\left(1-|a|^{2}\right) /|1-\langle a, z\rangle|^{2}\right)^{n+1}$.

See 2.2 of [5]. Now fix $w \in B_{n}$ and perform the change of variable $z \mapsto \varphi_{w}(z)$. Then we get

$$
\begin{aligned}
\int_{B_{n}} & \frac{\left(1-|z|^{2}\right)^{m}}{|1-\langle z, w\rangle|^{n+m+2}} d V(z) \\
& =\int_{B_{n}} \frac{\left(1-\left|\varphi_{w}(z)\right|^{2}\right)^{m}}{\left|1-\left\langle\varphi_{w}(z), w\right\rangle\right|^{n+m+2}}\left(\frac{1-|w|^{2}}{|1-\langle z, w\rangle|^{2}}\right)^{n+1} d V(z) .
\end{aligned}
$$

By Theorem 2.2.2 of [5],

$$
\begin{aligned}
1-\left|\varphi_{w}(z)\right|^{2} & =\frac{\left(1-|w|^{2}\right)\left(1-|z|^{2}\right)}{|1-\langle z, w\rangle|^{2}} \\
1-\left\langle\varphi_{w}(z), w\right\rangle & =1-\left\langle\varphi_{w}(z), \varphi_{w}(0)\right\rangle=\frac{1-|w|^{2}}{1-\langle z, w\rangle} .
\end{aligned}
$$

Putting these into the above integral, we get

$$
\int_{B_{n}} \frac{\left(1-|z|^{2}\right)^{m}}{|1-\langle z, w\rangle|^{n+m+2}} d V(z)=\frac{1}{1-|w|^{2}} \int_{B_{n}} \frac{\left(1-|z|^{2}\right)^{m}}{|1-\langle z, w\rangle|^{n+m}} d V(z) .
$$

By 1.4.10 of [5] (with $t=m, c=-1$ ), there exists a constant $C>0$ such that

$$
\int_{B_{n}} \frac{\left(1-|z|^{2}\right)^{m} d V(z)}{|1-\langle z, w\rangle|^{n+m}} \leq C
$$

for all $w$ in $B_{n}$. This completes the proof of Lemma 17 .

LEMMA 18. There exists a constant $C>0$ such that $\|f\|_{m, 1} \leq C\|f\|_{1}$ for all $f$ in $L_{a}^{1}\left(B_{n}\right)$.

ProOF. Given $f \in L_{a}^{1}\left(B_{n}\right)$, write

$$
f(z)=(n+1) \int_{B_{n}} \frac{\left(1-|w|^{2}\right) f(w)}{(1-\langle z, w\rangle)^{n+2}} d V(w) .
$$


Differentiating under integral gives

$$
\frac{\partial^{m} f}{\partial z^{\alpha}}(z)=(n+1)(n+2) \cdots(n+m+1) \int_{B_{n}} \frac{\bar{w}^{\alpha}\left(1-|w|^{2}\right) f(w)}{(1-\langle z, w\rangle)^{n+m+2}} d V(w) .
$$

This implies

$$
\left|\frac{\partial^{m} f}{\partial z^{\alpha}}(z)\right| \leq(n+1)(n+2) \cdots(n+m+1) \int_{B_{n}} \frac{\left(1-|w|^{2}\right)|f(w)|}{|1-\langle z, w\rangle|^{n+m+2}} d V(w)
$$

and

$$
\begin{aligned}
& \int_{B_{n}}\left(1-|z|^{2}\right)^{m}\left|\frac{\partial^{m} f}{\partial z^{\alpha}}(z)\right| d V(z) \leq(n+1)(n+2) \cdots(n+m+1) \\
& \cdot \int_{B_{n}}\left(1-|z|^{2}\right)^{m} d V(z) \int_{B_{n}} \frac{\left(1-|w|^{2}\right)|f(w)| d V(w)}{|1-\langle z, w\rangle|^{n+m+2}} \\
&=(n+1)(n+2) \cdots(n+m+1) \int_{B_{n}}\left(1-|w|^{2}\right)|f(w)| d V(w) \\
& \cdot \int_{B_{n}} \frac{\left(1-|z|^{2}\right)^{m}}{|1-\langle z, w\rangle|^{n+m+2}} d V(z) .
\end{aligned}
$$

By Lemma 17, there exists a constant $C_{1}>0$ such that

$$
\int_{B_{n}}\left(1-|z|^{2}\right)^{m}\left|\frac{\partial^{m} f}{\partial z^{\alpha}}(z)\right| d V(z) \leq C_{1} \int_{B_{n}}|f(z)| d V(z)
$$

for all $f$ in $L_{a}^{1}\left(B_{n}\right)$. This implies that

$$
\|f\|_{m, 1} \leq C\|f\|_{1}
$$

for some constant $C>0$ and all $f$ in $L_{a}^{1}\left(B_{n}\right)$.

LEMMA 19. There exists a constant $C>0$ such that $\|f\|_{1} \leq C\|f\|_{m, 1}$ for all $f$ in $L_{a}^{1}\left(B_{n}\right)$.

PROOF. Since $L_{a}^{2}\left(B_{n}\right)$ is dense in $L_{a}^{1}\left(B_{n}\right)$ and \|\|$_{m, 1}$ is dominated by \|\|$_{1}$ by Lemma 18, it suffices to find a constant $C>0$ such that $\|f\|_{1} \leq C\|f\|_{m, 1}$ for all $f$ in $L_{a}^{2}\left(B_{n}\right)$. Given $f$ in $L_{a}^{1}\left(B_{n}\right)$, the Hahn-Banach extension theorem gives a linear functional $F$ on $L_{a}^{1}\left(B_{n}\right)$ with norm 1 and $\|f\|_{1}=|F(f)|$. Since $L_{a}^{1}\left(B_{n}\right)^{*} \cong \mathscr{B}\left(B_{n}\right)$ and the Bloch norm is equivalent to the norm in $L_{a}^{1}\left(B_{n}\right)^{*}$, there must be a constant $C_{1}>0$ such that for any $f \in L_{a}^{2}\left(B_{n}\right)$, there exists $g \in \mathscr{B}\left(B_{n}\right)$ with $\|g\|_{\mathscr{B}} \leq C_{1}$ and

$$
\|f\|_{1}=|\langle f, g\rangle|=\left|\int_{B_{n}} f(z) \overline{g(z)} d V(z)\right| .
$$

By Theorem 6 and the proof of Lemma 8, we can write $g=g_{0}+\sum_{|\alpha|=m} S_{\alpha}^{*} g_{\alpha}$ with $\left\|g_{0}\right\|_{\mathscr{B}} \leq C_{2}\|g\|_{\mathscr{B}}$ and $\left\|g_{\alpha}\right\|_{\mathscr{B}} \leq C_{2}\|g\|_{\mathscr{B}}$. Mimicking the proof of Lemma 9 or Lemma 14, we can find a constant $C_{3}>0$ such that $\|f\|_{1} \leq C_{3}\|f\|_{m, 1}\|g\|_{\mathscr{B}} \leq$ $C_{1} C_{3}\|f\|_{m, 1}$ for all $f$ in $L_{a}^{2}\left(B_{n}\right)$. This completes the proof of Lemma 19.

LEMMA 20. If $\|f\|_{m, 1}<+\infty$, then $f \in L_{a}^{1}\left(B_{n}\right)$.

PROOF. It follows from Lemma 19 and the proof of Lemma 10.

Summarizing Lemmas 18-20, we have proved the following theorem. 
THEOREM 21. Suppose $m \geq 1$ and $f$ is holomorphic in $B_{n}$. Then $f \in L_{a}^{1}\left(B_{n}\right)$ iff all the functions $\left(1-|z|^{2}\right)^{m} \partial^{m} f(z) / \partial z^{\alpha}(|\alpha|=m)$ are in $L^{1}\left(B_{n}, d V\right)$. Moreover, the norms \|\|$_{m, 1}$ and \|\|$_{1}$ are equivalent on $L_{a}^{1}\left(B_{n}\right)$.

6. Results on the polydisc. In this section, we state the corresponding characterizations for the Bergman spaces $L_{a}^{p}\left(\mathbf{D}^{n}\right)(1 \leq p<+\infty)$ of the polydisc $\mathbf{D}^{n}$. To simplify notation and avoid technicality, we restrict out attention to the case $n=2$. For a holomorphic function $f\left(z_{1}, z_{2}\right)$ on $\mathbf{D}^{2}$, we write

$$
\begin{aligned}
T_{1} f\left(z_{1}\right) & =\left(1-\left|z_{1}\right|^{2}\right) \frac{\partial f}{\partial z_{1}}\left(z_{1}, 0\right), \quad z_{1} \in \mathbf{D}, \\
T_{2} f\left(z_{2}\right) & =\left(1-\left|z_{2}\right|^{2}\right) \frac{\partial f}{\partial z_{2}}\left(0, z_{2}\right), \quad z_{2} \in \mathbf{D}, \\
T f\left(z_{1}, z_{2}\right) & =\left(1-\left|z_{1}\right|^{2}\right)\left(1-\left|z_{2}\right|^{2}\right) \frac{\partial^{2} f}{\partial z_{1} \partial z_{2}}\left(z_{1}, z_{2}\right), \quad\left(z_{1}, z_{2}\right) \in \mathbf{D}^{2} .
\end{aligned}
$$

For $1 \leq p \leq \infty$ and $f$ holomorphic in $\mathbf{D}^{2}$, let

$$
\|f\|_{p}^{*}=|f(0,0)|+\left\|T_{1} f\right\|_{L^{p}(\mathbf{D}, d A)}+\left\|T_{2} f\right\|_{L^{p}(\mathbf{D}, d A)}+\|T f\|_{L^{p}\left(\mathbf{D}^{2}, d V\right)} .
$$

We characterize the Bergman spaces of the bidisc in the following theorem:

THEOREM 22. Suppose $1 \leq p<\infty$ and $f$ is holomorphic in $\mathbf{D}^{2}$, then $f \in$ $L_{a}^{p}\left(\mathbf{D}^{2}\right)$ iff the functions $\left(1-\left|z_{1}\right|^{2}\right) \partial f\left(z_{1}, 0\right) / \partial z_{1},\left(1-\left|z_{2}\right|^{2}\right) \partial f\left(0, z_{2}\right) / \partial z_{2}$, and $\left(1-\left|z_{1}\right|^{2}\right)\left(1-\left|z_{2}\right|^{2}\right) \partial^{2} f\left(z_{1}, z_{2}\right) / \partial z_{1} \partial z_{2}$ are in $L^{p}\left(\mathbf{D}^{2}, d V\right)$. Moreover, \|\|$_{p}$ and \|\|$_{p}^{*}$ are equivalent norms on $L_{a}^{p}\left(\mathbf{D}^{2}\right)$.

The proof of Theorem 22 follows essentially the same lines of the proof of Theorem 11. Lemma 8 is to be replaced by the decomposition

$$
f\left(z_{1}, z_{2}\right)=f\left(z_{1}, 0\right)+f\left(0, z_{2}\right)+z_{1} z_{2} h\left(z_{1}, z_{2}\right)
$$

when $f(0,0)=0$. There exists a constant $C>0$ such that $\|h\|_{p} \leq C\|f\|_{p}$ for all $f$, see Lemma 15 of [7]. Also $h$ in the above decomposition is unique. The bounded projection we use for Theorem 22 is given by

$$
Q f\left(z_{1}, z_{2}\right)=4 \int_{\mathbf{D} \times \mathbf{D}} \frac{\left(1-\left|w_{1}\right|^{2}\right)\left(1-\left|w_{2}\right|^{2}\right)}{\left(1-z_{1} \bar{w}_{1}\right)^{3}\left(1-z_{2} \bar{w}_{2}\right)^{3}} f\left(w_{1}, w_{2}\right) d V\left(w_{1}, w_{2}\right) .
$$

$Q$ is bounded on $L^{p}\left(\mathbf{D}^{2}, d V\right)$ for all $1 \leq p<\infty$; thus $Q^{*}$ is bounded on $L^{p}\left(\mathbf{D}^{2}, d V\right)$ for all $1<p \leq+\infty$, where

$$
Q^{*} f\left(z_{1}, z_{2}\right)=4\left(1-\left|z_{1}\right|^{2}\right)\left(1-\left|z_{2}\right|^{2}\right) \int_{\mathbf{D} \times \mathbf{D}} \frac{f\left(w_{1}, w_{2}\right)}{\left(1-z_{1} \bar{w}_{1}\right)^{3}\left(1-z_{2} \bar{w}_{2}\right)^{3}} d V\left(w_{1}, w_{2}\right) .
$$

Let $S$ be the operator defined by

$$
S f\left(z_{1}, z_{2}\right)=\overline{z_{1} z_{2}} f\left(z_{1}, z_{2}\right) .
$$

Then it is easy to check that for $f \in L^{p}\left(\mathbf{D}^{2}, d V\right)$, we have

$$
Q^{*} S f\left(z_{1}, z_{2}\right)=\left(1-\left|z_{1}\right|^{2}\right)\left(1-\left|z_{2}\right|^{2}\right) \frac{\partial^{2} f}{\partial z_{1} \partial z_{2}}\left(z_{1}, z_{2}\right)
$$

i.e., $Q^{*} S=T$. This corresponds to the formula $T_{\alpha}=m ! P_{m}^{*} S_{\alpha}$ in the proof of Lemma 7. We omit the details of the proof of Theorem 22. The space of analytic functions $f\left(z_{1}, z_{2}\right)$ on $\mathbf{D}^{2}$ with $\|f\|_{\infty}^{*}<+\infty$ was studied in [7]. It was shown there that the space is strictly larger than the Bloch space $\mathscr{B}\left(\mathbf{D}^{2}\right)$ of the bidisc. Interested readers can also refer to [7] for more information on the bidisc. 


\section{REFERENCES}

1. Sheldon Axler, Bergman spaces and their operators, Lecture Notes at the Indiana University Function Theoretic Operator Theory Conference, November 1985.

2. R. R. Coifman and R. Rochberg, Representation theorems for holomorphic and harmonic functions in $L^{p}$, Astérisque 77 (1980).

3. Peter Duren, Theory of $H^{p}$ spaces, Academic Press, New York, 1970.

4. F. Forelli and W. Rudin, Projections on spaces of holomorphic functions in balls, Indiana Univ. Math. J. 24 (1974), 593-602.

5. Walter Rudin, Function theory in the unit ball of $\mathbf{C}^{n}$, Springer, Berlin, 1980.

6. A. Zabulionsis, Differential operators in spaces of analytic function, Lithuanian Math. J. 24 (1984), 32-36.

7. Kehe Zhu, Duality and Hankel operators on the Bergman spaces of bounded symmetric domains, J. Funct. Anal. (to appear).

Department of Mathematics, University of Washington, Seattle, WashingTON 98195

Current address: Department of Pure Mathematics, University of Waterloo, Waterloo, Ontario N2L 3G1, Canada 